\title{
Bacterial Pattern in Acute Appendicitis
}

\author{
Abdussemee Abdurrazzaaq ${ }^{1}$, Oludolapo Afuwape ${ }^{1}$, Adeyinka Ademola ${ }^{1}$, Olusegun Fasina ${ }^{2}$ \\ 1. Department of Surgery, University College Hospital Ibadan, Oyo State, Nigeria. \\ 2. Department of Microbiology, University College Hospital Ibadan, Oyo State, Nigeria.
}

Correspondence to: Dr. Abdussemee Abdurrazzaaq. Email: razq2001@yahoo.com

\begin{abstract}
Background: Bacteria have been implicated in the aetiopathogenesis of acute appendicitis. Few reports exist on bacteriology of the disease in Africa. The knowledge of bacteria and antibiotic sensitivity pattern will assist in formulating treatment protocol for acute appendicitis in a resource-poor environment. Aim: This study sought to determine the bacterial and antibiotic sensitivity pattern in acute appendicitis in a local population in Nigeria. Methods: Patients with acute appendicitis presenting between November 2013 and October 2014 were studied. At surgery, $1 \mathrm{~cm}$ rim of appendix was cut from the base and transferred into the transport medium. The specimen was cultured for aerobic and anaerobic bacteria. Antibiotic sensitivity test was performed. Results: Sixty patients were recruited. Escherichia coli and Clostridium perfringes were the commonest aerobes and anaerobes respectively isolated. The aerobes were sensitive to third generation
\end{abstract}

cephalosporins $(78.1 \%-86.2 \%)$ and flouroquinolones $(75.9 \%-77.6 \%)$ and resistant to amoxycilin with clavulanic acid. The anaerobes were sensitive to metronidazole (96.5\%). Conclusion: The commonest aerobic and anaerobic bacteria isolated are Escherichia coli and Clostridium perfringes respectively. The aerobes are sensitive to flouroquinolones and third generation cephalosporin. Anaerobes are sensitive to metronidazole. Combination of Ciprofloxacin and Metronidazole as prophylactic antibiotics or as eventual treatment for catarrhal appendicitis is recommended.

Key words: Bacteria, Appendicitis, Antibiotics

Ann Afr Surg. 2018; 15(1):8-13

DOI:http://dx.doi.org/10.4314/aas.v15i1.3

(C) 2018 Author. This work is licensed under the Creative Commons Attribution 4.0 International License

\section{Introduction}

Acute appendicitis is one of the commonest causes of surgical acute abdomen. The incidence is high in Western countries especially Europe and America. In England, the incidence is $100-130$ per 100,000 population $(1,2)$. In Africa and many other developing countries, appendicitis was rare in the early half of twentieth century (3). Appendicitis is common in adolescent and young adults although it can occur in any age group. Recently, the incidence of appendicitis has increased mainly due to dietary modification to the Western type (1). The incidence of acute appendicitis in one of the African cities (Soweto in South Africa) rose from 8.2 per 100,000 populations in 1987 to 9.5 per 100,000 populations in 1994(2). It accounts for $15 \%$ $40 \%$ of all emergency surgery done in most centers in Nigeria (1), and the cost of treatment which is unprepared for is largely provided by the patients or relatives.

\section{Etiology and Pathogenesis}

Increased consumption of refined carbohydrate with decreased dietary fibre has been linked to acute appendicitis(2). Luminal obstruction is one of the major causes of acute appendicitis. The fecolith is the commonest cause of the obstruction. Other causes of luminal obstruction include parasites, lymphoid hyperplasia, seeds or neoplasm. The obstruction of appendicial lumen produces a closed loop obstruction. There is continued secretion, stasis and bacterial overgrowth as well as intraluminal distension and increased wall pressure. Distension of the appendix stimulates nerve endings of visceral afferent stretch fibers, producing vague, dull, diffuse pain in the mid- 
abdomen or lower epigastrium, nausea and vomiting. With progressive distension, the venous pressure is exceeded; the capillaries and the venules become occluded. The arterial blood flow persists, leading to vascularcongestion, engorgement and local ischemia. Consequently, arteriolar occlusion occurs, with progressive infarction and subsequent gangrene and perforation. Serosal involvement in the inflammation causes localized pain in the right lower quadrant( ). Consequently, arteriolar occlusion occurs, with progressive infarction and subsequent gangrene and perforation. Serosal involvement in the inflammation causes localized pain in the right lower quadrantLuminal obstruction seems not to explain the aetiology of acute appendicitis in all cases because some patients with appendicitis have patent lumen on radiologic, gross and histological examination. Luminal obstruction had been reported in only $30 \%-40 \%$ of removed inflamed appendices in one study (4).Acute appendicitis may also be of catarrhal inflammatory origin. In this case, there is no luminal obstruction but there is infiltration of the appendicial submucosa and muscularis propria by inflammatory cells and bacteria. This type of appendicitis usually resolves with administration of antibiotics. Therefore, bacteria have been implicated in both obstructive and catarrhal appendicitis.

The bacterial flora in the appendix consists of a mixture of facultative aerobes and obligate anaerobes $(4,5)$. These are similar to those found in the colon. The commonest bacteria isolated in many reported cases are gram negative bacilli Bacteroides fragilis and Escherichia coli $(5,6)$. Bacteroides fragilis have been isolated in over $80 \%$ of cases (5). Gram positive cocci have also been isolated in the appendix. Acute appendicitis is a polymicrobial infection, up to 14 bacteria isolates have been reported in one study(7). Antibiotics play important roles as peri-operative prophylaxis or main treatment in catarrhal appendicitis whereby there may be resolution of the disease process with antibiotic use. Knowledge of the bacteria and their sensitivity pattern to antibiotics will help guide the choice of prophylactic antibiotics and thus control post operative wound infection.
In the era of medical treatment for selective cases of acute appendicitis, the bacterial sensitivity and resistant pattern will determine the choice of antibiotics combination especially in our locality. This study was designed to determine the bacterial and antibiotic sensitivity pattern in acute appendicitis.

\section{Methods}

The study was prospective, done over a period of 12 months (November, 2013 to October 2014). All patients aged above 15 years presenting through Emergency Department and Surgical Outpatient Clinic of the University College Hospital Ibadan Nigeria with clinical diagnosis of uncomplicated acute appendicitis were recruited for the study. The proforma for the study was given and explained in detail to all doctors in each unit of the general surgery division. The patients were clerked and the diagnosis was made by the doctors in the units admitting the patients. Informed consent for the surgery and the study was obtained from the patients. The patients' biodata were obtained along with detailed clinical presentation. The routine protocol for treatment of acute appendicitis was commenced. The protocol included admitting the patient, secure intravenous access while at the same time taking blood samples for full blood count, electrolytes, urea and creatinine; urine analysis, intravenous fluid and antibiotics, and consent for the operation. The surgery was performed by either consultant or senior registrar. The surgical approach was through a skin crease (Lanz) incision. A routine appendicectomy was done. A 1centimeter rim of tissue from the proximal end of the appendicial specimen of the patients was cut by the surgeon immediately after the specimen was severed from the caecum. The sample was immediately transferred into prepared sterile Robertson Cooked Meat Medium in sterile glass universal bottles properly labelled for transfer to the laboratory for culture. Part of the sample was cultured aseptically on blood agar, chocolate agar and MacConkey agar respectively and immediately incubated at $37^{\circ} \mathrm{C}$ for 24 hours to isolate aerobic microorganisms. The remaining part was cultured aseptically on blood agar, chocolate agar and anaerobic agar respectively; and immediately transferred into an anaerobic jar with a fixed anaerobic kit and controlled organisms. They were then incubated 
at $37^{\circ} \mathrm{C}$ for five days. All the bacterial growths were examined processed and confirmed using API 20 (Biomeriux Pioneering Diagnostics) for proper speciation in accordance with the manufacturer's instruction. All the confirmed isolates were tested for antibiotic sensitivity testing using Muller Hinton agar plate for aerobic bacteria and blood agar plate for anaerobic bacteria. The choice of antibiotics tested for was based on the common antibiotics used in our environment both within and outside the hospital. Preoperative, intra-operative and culture data were collected using data collection proforma. The parameters collected were Biodata, clinical features such as abdominal pain, fever, anorexia, vomiting, nausea, right lower abdominal tenderness and rebound tenderness. Others were full blood count, state of the appendix at surgery whether it is normal, inflamed or ruptured; specimen culture results and antibiotics sensitivity.Data collected were computed and analyzed using Statistical Package for Social Sciences (SPSS) version 21. Results were presented using tables. Categorical data were summarized by frequencies and percentages. The bacterial and their antibiotics sensitivity patterns were noted.

Ethical approval for the study was sought and obtained from the institutional review board (IRB) - University of Ibadan/University College Hospital Ethical Committee

Exclusion Criteria:

The patients below the age of 15 years were not enrolled in to the study as they belong to the pediatric age group. Other patients excluded were those that had peritonitis from ruptured appendix and those that had incidental appendicectomy whereby appendix was removed during laparotomy for indication other than acute appendicitis.

\section{Results}

Sixty patients were recruited in the study, $26(43.3 \%)$ males and $34(56.7 \%)$ females with a male: female ratio of $1: 1.3$. The ages ranged between 15 and 52 years with a median age of 25 years. All the patients had abdominal pain as presenting complaint, 45 patients (75\%) had anorexia with the abdominal pain and only 9 patients $(15 \%)$ had fever. All the patients had right iliac fossa tenderness, 43 patients $(71.3 \%)$ had rebound tenderness and only 9 patients (15\%) had pyrexia at the time of presentation (Table 1).

Table 1: Pattern of symptoms and signs of acute appendicitis

\begin{tabular}{|l|l|l|}
\hline Clinical features & Frequency & Percent \\
\hline Abdominal pain & 60 & 100 \\
\hline Anorexia & 45 & 75 \\
\hline Vomiting & 36 & 60 \\
\hline Pyrexia & 9 & 15 \\
\hline Right iliac fossa & 60 & 100 \\
\hline Rebound tenderness & 43 & 71.1 \\
\hline
\end{tabular}

All the patients had pre-operative antibiotics and majority of them (95\%) had combination of ciprofloxacin and metronidazole antibiotics.

The appendix was grossly inflamed in 55 patients (91.6\%) including 5 patients with ruptured appendix while remaining 5 patients $(8.3 \%)$ had normal appendix. There was significant association between the inflamed appendix and leucocytosis $(\mathrm{x} 2=9.02$, $\mathrm{p}$ value $=0.01)$.

Majority of the aerobic bacteria isolated were Escherichia coli (50\%), Kleibsiella spp (15\%) and Proteus spp $(8.3 \%)$. No bacterial growth was observed in 2 specimens. The aerobic bacterial isolates are shown in Table 2. Most of the anaerobic bacteria isolated were Clostridium perfringes (51.7\%). Others were Anaerococcus prevotii (13.3\%) and Actinomyces isrealis $(10 \%)$. The arrays of anaerobic bacterial isolates are shown in Table 2. The bacteria profile of the normal appendices were not different from those from inflamed or ruptured ones, with Stapphylococcus aureus (40\%) and $\mathrm{C}$ perfringes $(60 \%)$ being the most common aerobic and anaerobic bacteria respectively isolated. Others aerobes found were E coli (20\%), Kleibsiella spp (20\%), and Proteus spp (20\%). Anaerococcus prevotii was the second anaerobe isolated.

There was no significant association between the types of bacterial isolates and symptoms such as anorexia, pyrexia; or leucocyte count. Majority of the aerobic bacteria were sensitive to ciprofloxacin $(75.9 \%)$, sparfloxacin $(77.6 \%)$ and ceftriaxone $(86.2 \%)$. Seventyfour percent of the bacteria were resistant to amoxyclav (amoxycilin + clavulanic acid) and almost all had resistance to tetracycline $(96.6 \%)$. The anaerobic bacteria were sensitive to metronidazole $(96.5 \%)$. The 
Table 2: Bacterial Isolates from Appendicectomy Specimen

\begin{tabular}{|l|l|l|l|l|l|}
\hline Aerobic Bacteria & No. & $\mathbf{\%}$ & Anaerobic Bacteria & No & $\mathbf{\%}$ \\
\hline Acenetobacter baumanii & 4 & 6.7 & Actinomyces Israelis & 6 & 10.0 \\
\hline Escherichia. Coli & 26 & 43.3 & Anaerobic staphylococcus & 1 & 1.7 \\
\hline Escherichia coli, Acinobacter baumannii & 3 & 5.0 & Anaerococcus prevotii & 8 & 13.3 \\
\hline Escherichia coli, Kleibsiella spp & 1 & 1.7 & Clostridium butrilimo & 1 & 1.7 \\
\hline Enterobacter spp & 1 & 1.7 & Clostridium perfringes & 31 & 51.7 \\
\hline Enterobacter spp, Staphylococcus aureus & 1 & 1.7 & Clostridium tetanii & 2 & 3.3 \\
\hline Kleibsiella spp & 8 & 13.3 & Peptococcus anaerobius & 4 & 6.7 \\
\hline Proteus spp & 5 & 8.3 & Peptococcus niger & 2 & 3.3 \\
\hline Pseudomonas spp & 4 & 6.7 & Porpyromonas spp & 1 & 1.7 \\
\hline Staphylococcus aureus & 4 & 6.7 & Propionibacterium acnes & 1 & 1.7 \\
\hline Streptococcus faecalis & 1 & 1.7 & no growth & 3 & 5.0 \\
\hline no growth & 2 & 3.3 & & & \\
\hline Total & 60 & 100.0 & Total & 60 & 100 \\
\hline
\end{tabular}

Table 3: Antibiotic Sensitivity of the Aerobic Bacteria

\begin{tabular}{|l|l|l|}
\hline \multicolumn{1}{|c|}{ Antibiotics } & No & \% \\
\hline Ciprofloxacin & 44 & 75.9 \\
\hline Sparfloxacin & 45 & 77.6 \\
\hline Ceftaxidime & 46 & 78.1 \\
\hline Ceftriaxone & 50 & 86.2 \\
\hline PefloxacineAmoxycillin/Cla & 44 & 75.9 \\
\hline vulanic acid*** & 15 & 25.9 \\
\hline Cefuroxime & 44 & 75.9 \\
\hline Tetracycline & 2 & 3.4 \\
\hline
\end{tabular}

antibiotics sensitivity patterns for aerobic bacteria are shown in Table 3. Thirty-six patients $(60 \%)$ were discharged on the post- operative day 3 , seventeen patients $(28.2 \%)$ on post-operative day 2 and two patients on the first day after surgery.

\section{Discussion}

Appendicitis is one of the commonest causes of surgical acute abdomen presenting to the surgeons. The rate of appendicitis is slightly higher in female in this study with a male to female ratio of $1: 1.3$. The reason for this is not clear but this is similar to a study in Port Harcourt, Nigeria by Mangete (8) et al. Other studies showed higher incidence in male (9). Most of the patients in this study were between 15 and 30 years $(68.3 \%)$. This is similar to findings from other studies $(4,9)$ where peak incidence from 10 to 30 years was found. All the patients had right iliac fossa pain at presentation. Anorexia has been noted to be a constant symptom of acute appendicitis and its absence makes the diagnosis of acute appendicitis questionable (5). This is not so in this study as only $75 \%$ had anorexia. A lower occurrence of anorexia (43\%) has been reported by Sheu et al (11).

Right iliac fossa tenderness was a common sign in all the patients, rebound tenderness was found in $71.3 \%$ of patients and pyrexia in only $15 \%$. This is similar to the findings in other study (11) where rebound tendernesswas found only in $72.9 \%$ of patients. Most patients with uncomplicated acute appendicitis seldom present with fever. A fever of greater than $38.2^{\circ} \mathrm{C}$ may be a pointer of complicated appendicitis (5). Pyrexia was found to be significantly associated with leucocyte count. All the patients had antibiotics either before presentation or in the Emergency Department and at induction of anesthesia. Ciprofloxacin and metronidazole has been the practice in the Emergency Department for any patient presenting with acute abdomen or trauma. This is based on the pharmacology of ciprofloxacin as a broad spectrum antibiotic that is effective against wide range of Gram positive and negative bacteria (15). Metronidazole choice is also based on the pharmacology of the drug as being very effective against most anaerobes (16). Pre-operative (or prophylactic) antibiotics aim at reducing post operative surgical site infection, and not directly targeting at the gut bacteria. The antibiotics did not affect the bacteria culture as only two specimens yielded no growth in this study. Gross examination of the specimen showed inflamed appendix in most of the specimen $(83.3 \%)$ and ruptured appendix in $8.3 \%$. The patients with intra- 
operative finding of ruptured appendix were included in the study as they did not have generalized peritonitis and no frank pus in the right iliac fossa. The grossly normal appendix rate of $8.3 \%$ (which hitherto might be considered as negative appendicectomy though histology was not focused on in this study), is comparable to the rate of $6.5 \%$ and $15.3 \%$ recorded by Christian (17) et al and Flum (18) et al respectively. This shows that clinical evaluation alone or together with complete blood count may be highly reliable.

Escherichia coli and Clostridium perfringes were the predominant aerobic and anaerobic bacteria respectively isolated from this study. E. coli has been reported to be the most common aerobic bacteria in the lumen of appendix from many studies but the commonest anaerobe (in fact the commonest bacteria) reported is Bacteroides fragilis. (19) In contrast to this, B. fragilis was not isolated in this study. The bacterium has been described as a fastidious organism is difficult to cultivate in vitro (20). Its growth is usually suppressed or inhibited in the presence of other micro-organisms. A special culture medium [Bacteroides fragilis BileEsculin Agar (21)] which contains bile salt, esculin and gentamycin in a trypticase soy agar base may be needed to isolate $\mathrm{B}$. fragilis. The absence of $\mathrm{B}$. fragilis from the isolates might also not be unconnected with the culture techniques. Other obligate anaerobes that are fastidious might have also been missed; thereby making Clostridium perfringes the commonest anaerobe isolated in the study. Lau (16) et al found $B$. melanogenicus and fusobacterium spp as next common anaerobes after $B$. fragils followed by $C$. perfringes. There is no association between clinical features like anorexia, pyrexia, white cell count and the type of bacteria isolated from the specimens.

Majority of the bacterial isolates were sensitive to fluoroquinolones (ciprofloxacin, sparfloxacin and pefloxacin) and third generation cephalosporin. The aerobes have high resistance to penicillin containing drugs and tetracycline. Anaerobic bacteria are most sensitive to metronidazole. Bacterial sensitivity to flouroquinolones especially ciprofloxacin and sparfloxacin is comparable to third generation cephalosporin like ceftriaxone and ceftaxidime. Therefore a combination of ciprofloxacin and metronidazole will suffice as prophylactic antibiotics in uncomplicated appendicitis. A dose of branded ceftriaxone costs about five times the price of ciprofloxacin. Consequently this reduces the cost of antibiotics. The antibiotics may also be the only treatment required particularly for the patients with catarrhal inflammatory appendix who showed improvement and resolution of the features of acute appendicitis upon commencement of antibiotics. This has been reported to be safe and comparable to appendicectomy (22). Malik (23) et al in his study concluded that antibiotic treatment in the patients with acute appendicitis is quite effective, and these patients may not need surgery. Intravenous administration of the drugs for 48 hours before changing to oral medication is recommended (24). There are some limitations to the study. Bacteroides fragilis and other Bacteroides species were not isolated from the culture of the appendicectomy specimen. Culture medium (Bacteroides fragilis Bile-Esculin Agar) specific for the organisms was not used further when unable to culture them from the media earlier used

\section{Conclusion}

Appendicitis is a common surgical disease especially among the young adults with equal affliction of the sexes. Clinical evaluation can accurately give the diagnosis in over $90 \%$ of cases. Clostridium perfringes and Escherichia coli are the most common bacterial isolates and are sensitive to flouroquinolones, third generation cephalosporins and metronidazole.

\section{Recommendation}

In areas with limited facilities and expertise patients with a clinical diagnosis of acute appendicitis should be commenced on antibiotics. The combination of ciprofloxacin and metronidazole for prophylaxis or treatment for catarrhal appendicitis is advocated. A clear referral criterion is needed in facilities without surgical capacity.

\section{Conflict of Interest}

No conflict of interest in this study.

\section{References}

1. Walker ARP, Segal I. Appendicitis: An African Perspective. J R Soc Med. 1995; 88:616-9.

2. Burkitt DP. The Aetiology of Appendicitis. Br J Surg 1971; 58:695. 
3. Alatise OI, Ogunweide T. Acute Appendicitis: Incidence and Management in Nigeria. IFEMED Journal 2008; 14 (1): 66-70.

4. Carr NJ. The Pathology of Acute Appendicitis. Ann Diagn Pathol. 2000;4(1):46-58.

5. Leigh DA, Kate S, Edelgard N. Bacterial Flora of the Appendix Fossa in Appendicitis and Postoperative Wound Infection. J. Clin. Path 1974; 27: 997-1000.

6. Soffer D, Zait S, Klausner J, et al. Peritoneal Cultures and Antibiotic Treatment in Patients with Perforated Appendicitis. Eur J Surg 2001 Mar; 167(3):214-6.

7. Rautio M, Saxén H, Siitonen A, et al. Bacteriology of Histopathologically Defined Appendicitis in Children. Pediatr Infect Dis J 2000; 19(11):1078-83.

8. Mangete ED, Kombo BB. Acute Appendicitis in Port Harcourt, Nigeria. Orient J Med 2004; 16:1-3.

9. Oguntola AS, Adeoti ML, Oyemolade TA. Appendicitis: Trends in Incidence, Age, Sex, and Seasonal Variations in South-Western Nigeria. Ann Afr Med 2010; 9(4):213-7.

10. Ajao OG. Appendicitis in a Tropical African Population. J Natl Med Assoc 1979; 71: 997-9

11. Sheu BF, Chiu TF, Chen JC, et al. Risk Factors Associated with Perforated Appendicitis in Elderly Patients Presenting with Signs and Symptoms of Acute Appendicitis. ANZ J Surg. 2007;77(8):662-6.

12. Vance-Bryan K, Guay DR, Rotschafer JC. Clinical Pharmacokinetics of Ciprofloxacin. Clin Pharmacokinet 1990; 19(6): 434-61.

13. Brogden RN, Heel RC, Speight TM, et al. Metronidazole in Anaerobic Infections: A Review of its Activity, Pharmacokinetics and Therapeutic Use- Drugs 1978; 16: 387-417.

14. Christian F, Christian GP. A Simple Scoring System to Reduce the Negative Appendicectomy Rate. Ann R Coll Surg Engl. 1992; 74(4): 281-5.

15. Flum DR, Koepsell T. The Clinical and Economic Correlates of Misdiagnosed Appendicitis: Nationwide Analysis. Arch Surg. 2002; 137(7):799804.

16. Lau WY, Teoh-Chan CH, Fan ST, et al. The Bacteriology and Septic Complication of Patients with Appendicitis. Ann Surg 1984; 200: 576-81.
17. Berg JO, Nord CE, Wadström T. Formation of Glycosidases in Batch and Continuous Culture of Bacteroides Fragilis. Appl Environ Microbiol. 1978; 35(2): 269-73.

18. Livingston SJ, Kominos SD, Yee RB. New Medium for Selection and Presumptive Identification of the Bacteroides Fragilis Group. J Clin Microbiol. 1978; 7(5): 53.

19. Varadhan KK, Neal KR, Lobo DN. Safety and Efficacy of Antibiotics Compared with Appendicectomy for Treatment of Uncomplicated Acute Appendicitis: Meta-Analysis of Randomized Controlled Trials. BMJ 2012; 344: E2156.

20. Malik AA, Bari S. Conservative Management of Acute Appendicitis. J Gastrointest Surg 2009; 13:966-70.

21. Wojciechowicz KH, Hofkamp HJ, Van Hulst RA. Conservative Treatment of Acute Appendicitis: An Overview. Int Marit Health 2010; 61(4): 265-72. 